\title{
LA RENTABILIDAD SOCIAL DE LA INVESTIGACIÓN AGRÍCOLA GUBERNAMENTAL EN GRANOS BÁSICOS: EL CASO DEL MAÍZ EN GUATEMALA, 1973-901
}

\author{
Mamerto Reyes ${ }^{2}$
}

\begin{abstract}
RESUMEN
La rentabilidad social de la investigación agrícola gubernamental en granos básicos: el caso del maíz en Guatemala, 1973-90. En este trabajo se evalúa la rentabilidad social de la investigación agrícola en maíz desarrollada por el Instituto de Ciencia y Tecnología Agrícolas (ICT A) de 1973 a 1990. Se usa un modelo econométrico del mercado nacional de maíz en Guatemala. Como beneficios sociales se toman los incrementos en los excedentes de productores y las divisas ahorradas por evi tar importaciones de maíz, resultantes de la investigación en este cereal. En la evaluación se obtuvo una Relación B/C de 6,06 y una TIR de $96.95 \%$, los cuales indican que la investigación agrícola en maíz ha sido una actividad altamente rentable para la sociedad nacional.
\end{abstract}

\begin{abstract}
The social rentability of government agricultural researh on basic grains: the Guatemala maize case, 197390. In this paper we evaluate the social rentability of maize agricultural research conducted at the Institute of Agricultural Science and Technology (ICTA), from 1973-90. We used an econometric model ofthe national maize market in Guatemala. As social benefits we take into account the increases in the surpluses ofproducers and the savings obtained by notimporting maize. In the evaluation we obtained a B/C relationship of 6.06 and a TIR of $96.95 \%$, which indicate that maize agronomic research has proved to be a highly profitable activity for this society.
\end{abstract}

\section{INTRODUCCIÓN}

La importancia de la investigación agrícola para apo-yar la seguridad alimentaria ha sido recalcada por las implicaciones de política de algunos estudios realizados en países subdesarrollados, tales como los trabajos sobre la respuesta a los precios del excedente comercial de arroz en Filipinas y Taiwan (Taquero et al, 1975 y Chinn, 1976), en los cuales se encontró que las medidas de política que afectan los rendimientos, tales como la investigación agrícola y las obras de irrigación, son más eficientes para incrementar el excedente comercial que la política de precios. Por otra parte, estudios realizados en países industrializados (Griliches, 1964 y Evenson, 1967), han demostrado la relevancia de la investigación agrícola en el crecimiento de la productividad agropecuaria. Para el caso de Estados Unidos, Evenson (1978) indica que el creci-miento de la productividad agropecuaria en los últimos cien años, en buena parte es una consecuencia directa de la investigación agrícola.
En Guatemala, Reyes (1995), determinó la relevancia de la investigación agrícola en maíz, en el sostenimiento de la autosuficiencia de este cereal en niveles cercanos al $100 \%$ durante el período 1975-90.

Para la evaluación de la rentabilidad social en la literatura se encuentran tres enfoques alternativos, a saber: el enfoque de los excedentes económicos (EEE), el enfoque de la función de producción (EFP) y el enfoque de las ganancias privadas de los agricultores (EGA).

Con el EEE se busca determinar el cambio en los excedentes de productores y consumidores que resulta del desplazamiento de la oferta que ocasiona la tecnología generada por la investigación agrícola. Este cambio en el excedente económico constituye la ganancia social, la cual al analizarse en conjunto con los costos del programa de investigación permite determinar su rentabilidad. Entre los trabajos con este enfoque se pueden citar Scobie y Posada (1976) y Evenson y Flores (1978).

\footnotetext{
1 Presentado en la LXII Reunión Anual del PCCMCA en El Salvador, 1996.

2 Instituto de Ciencia y Tecnología Agrícolas (ICTA), Guatemala.
} 
Con el EFP se busca determinar la productividad marginal de la inversión en investigación. Este enfoque fundamentalmente se basa en la introducción del conocimiento técnico como un factor adicional en la función de producción. Esta introducción se ha hecho a través de diversas variables proximales (proxis), gastos de investigación y extensión, publicaciones científicas en ciencias agrícolas y científicos trabajando en instituciones de investigación agrícola (Norton y Davis, 1981).

El EGA es un enfoque usado para evaluar retornos sociales a nivel local, comunal o subregional. Este enfoque parece haber surgido como resultado de las dificultades encontradas en la aplicación de los dos enfoques anteriores en áreas relativamente pequeñas. Con el EGA, las ganancias sociales están representadas por el incremento en las ganancias de los productores resultante de la adopción de tecnología. Para derivar los incrementos en las ganancias en el tiempo se requieren estudios longitudinales de adopción de tecnología, los cuales permiten derivar el efecto de la adopción en los rendimientos, producción, costos e ingresos de los agricultores. Este enfoque ha sido usado por Martínez y Saín (1983) y por Saín y Matute (1993).

El presente trabajo es parte de una serie de estudios que se están realizando en el ICT A para determinar la importancia económica de la investigación agrícola para Guatemala. En este trabajo se busca determinar la rentabilidad social de la investigación agrícola en maíz de los primeros 18 años de trabajo del ICTA, período 1973-90.

\section{MARCO CONCEPTUAL}

\section{El Mercado}

El mercado de maíz en Guatemala es un mercado abierto y deficitalio, es decir, en él participa el sector externo y la producción interna es insuficiente para atender a la demanda.

Un modelo de este mercado se representa en la Figura 1. En este modelo, QSR1 es la oferta en el mercado rural o mercado del productor en las condiciones previas al cambio tecnológico, QSR2 es la oferta en el mercado rural considerando el nuevo estado tecnológico, QDC es la demanda en el mercado al consumidor y QSC es la oferta en el mercado al consumidor. La oferta en este nivel de mercado es la suma de la producción interna y las importaciones menos las exportaciones. Por otra parte, PINT es el precio vigente en el mercado internacional más los costos necesarios para colocar el producto en la frontera y PR es el precio en el mercado rural.

En el mercado de maíz en Guatemala, como resultado de la apertura económica, el PR es igual al PINT, resulta rentable la importación de productos, hasta el nivel en que los precios internos se igualan a los externos. Por otra parte, el nivel de precios al productor logrado con la importación de producto, dadas las funciones de producción existentes en el país, conduce a niveles de producción incapaces de satisfacer al consumo interno. Pero, como consecuencia del mejoramiento de la

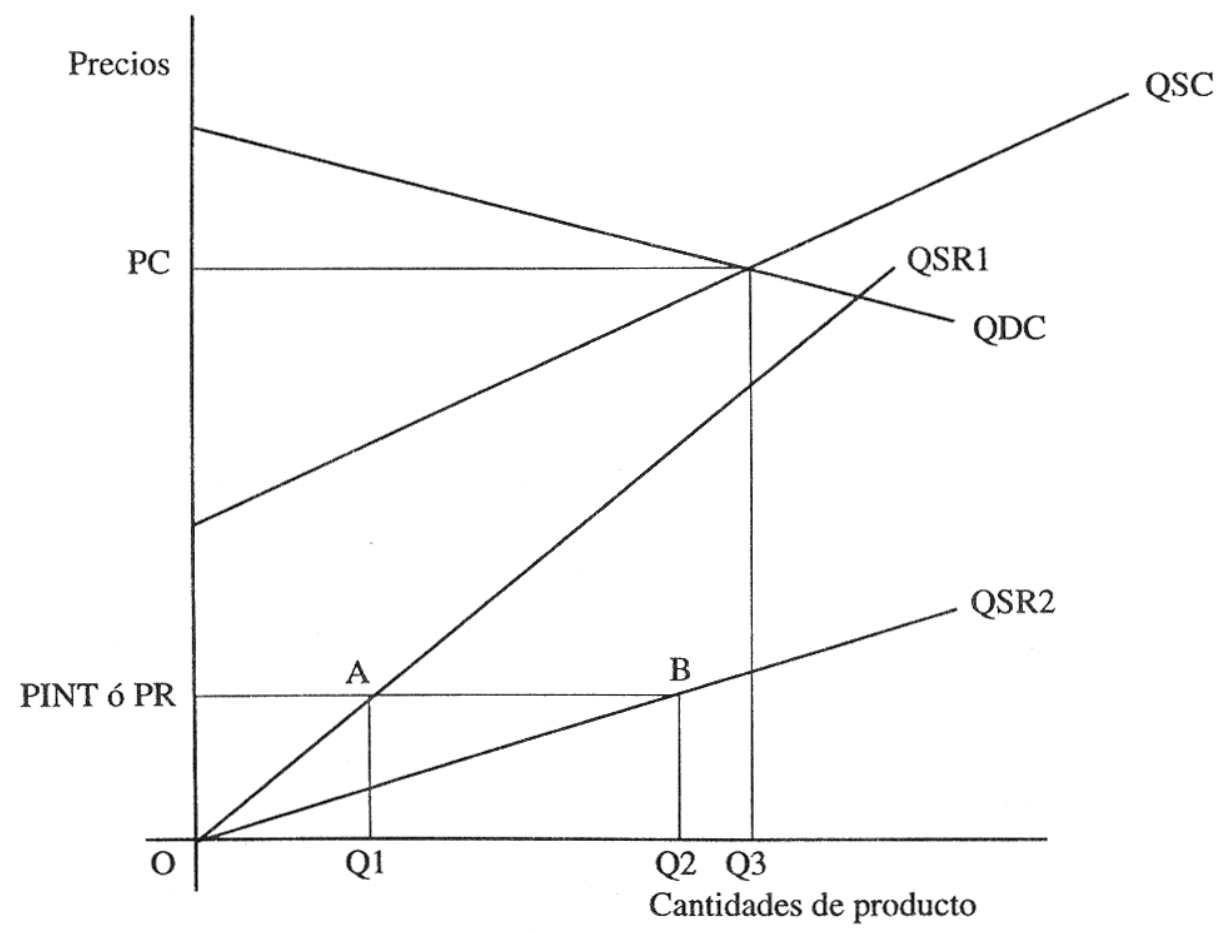

Fig. 1. Desplazamiento de la oferta en un mercado deficitario y en una economía abierta. 
eficiencia productiva, resultante de la investigación agrícola, la capacidad de los productores nacionales, para atender al consumo, se ve aumentada.

\section{Los beneficios sociales}

En mercados de esta naturaleza los consumidores demandan la misma cantidad de producto indistintamente de la tecnología que usen los productores. En la Figura 1 se muestra que la cantidad consumida de maíz es Q3.

Si los agricultores continuan empleando la tecnología tradicional, al precio PR pueden ofrecer Q1, por lo que para proveer a los consumidores, el país debe importar la diferencia Q3 - Q1. Si los agricultores usan la tecnología mejorada, al precio PR pueden ofrecer Q2, lo cual reduce las importaciones de arroz en Q2 - Q 1. De donde se deduce que el país ahorra un valor de PINT*(Q2 - Q 1), el cual es un primer beneficio que gana la sociedad como resultado del cambio tecnológico.

Ahora, ¿Qué ganan los agricultores con este proceso? En mercados con naturaleza similar al del maíz en Guatemala, el cambio tecnológico conduce a incrementar los excedentes de los productores.

El excedente del productor es la suma agregada de la diferencia que existe entre el precio de equilibrio y el costo marginal de producir cada una de las cantidades que conforman la función de oferta hasta llegar al punto en que esta diferencia es cero.

En la Figura 1, bajo condiciones de tecnología tradicional, el excedente de los productores lo representa el área OAPR. Bajo condiciones de tecnología mejora$\mathrm{da}$, el excedente de los productores es el área OBPR. De donde se deduce que como resultado del cambio tecnológico, el excedente de los productores se incrementa en $\mathrm{OAB}$, el cual es el segundo beneficio que gana la sociedad con este proceso.

\section{MODELO EMPÍRICO}

\section{Modelo del mercado de maíz}

El prototipo descrito en la Figura 1, se modeló econométricamente de la manera siguiente:

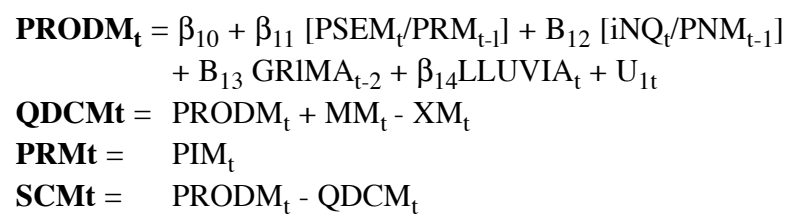

en donde:

U Es el término aleatorio de error

PRODM Oferta de maíz en el mercado al productor (miles t);

QDCM Cantidad demandada de maíz en el mercado de consumo (miles $\mathrm{t}$ );

PRM Precio real de maíz al productor (Quetzales/t);

SCM Saldo de comercio de maíz (miles t);

$\mathbf{P R M}_{\mathbf{t}} \quad$ Precio real esperado de maíz al productor. Precio del año anterior (Quetzales/t);

PNM $_{\text {t-1 }}$ Precio nominal esperado de maíz al productor. Precio del año anterior (Quetzales $/ \mathrm{kg}$ );

PSEM Precio real de la semilla mejorada de maíz (Quetzales/t);

iNQ Costo nominal de los intereses de un Quetzal (Quetzales)

GRIMA $_{\mathbf{t}-2}$ Conocimiento técnico o factor de cambio tecnológico. Medido a través de los gastos reales de investigación en maíz acumulados con dos años de rezago (miles Quetzales);

LLUVIA Precipitación pluvial anual (mm);

MM Importaciones de maíz (Quetzales/t);

XM Exportaciones de maíz (miles t);

PIM Precio CIF real de importación de maíz (Quetzales/t).

\section{Modelos para medir los beneficios del cambio tecnológico}

Para evaluar los beneficios del cambio tecnológico tradicionalmente se sigue el método del "con y el sin", el cual no es más que un formato para hacer comparaciones entre lo que pasa con el proyecto y lo que pasaría sin el proyecto. En este trabajo, la adaptación del "con y el sin" se hizo a través de la comparación de lo que pasó con los programas de investigación y lo que hubiese pasado si estos hubiesen sido interrumpidos. Para cada año de la serie estudiada, se comparó lo que ocurrió ese año con lo que hubiese ocurrido si el programa hubiese sido interrumpido el año anterior. Este procedimiento evaluativo permitió estimar la serie de beneficios sociales marginales resultante de las decisiones de continuar anualmente los programas de investigación.

Creemos que la aplicación ortodoxa del "con y el sin" en un horizonte de tiempo de la longitud del evaluado en este estudio (18 años), hubiese conducido a una sobrevaluación de los beneficios, fundamentalmente debido a que el escenario "sin proyecto" no resulta apegado a la realidad, pues en el largo plazo, la ausencia de un programa nacional de investigación agrícola motiva a los agricultores a que busquen otras alternativas para elevar la productividad y a que las empresas privadas nacionales e internacionales las ofrezcan. 
Para hacer operativa la medición de los beneficios sociales del cambio tecnológico en cada uno de los cultivos estudiados, con las funciones de oferta de los modelos econométricos, se formularon funciones para los escenarios con y sin cambio tecnológico correspondientes a nuestra adaptación del "con y el sin". De acuerdo con la formulación del modelo econométrico, el efecto de la investigación agrícola en el t-ésimo año lo representa GRIMA $\mathrm{t}_{-2}$, de donde se deduce que la ausencia del efecto de la investigación agrícola en el t-ésimo año lo representa GRIMA $\mathrm{t}_{\mathrm{t}-3}$. Siguiendo este argumento, las funciones de oferta con cambio tecnológico consideraron la variable GRIMA $_{\mathrm{t}-2} \mathrm{y}$ las funciones sin cambio tecnológico consideraron a GRIMA $\mathrm{G}_{\mathrm{t}-3}$.

Para estimar los beneficios anualmente se emplearon funciones de la forma:

$$
\begin{array}{ll}
\text { PRODM }_{\mathrm{t}}= & \mathrm{f}_{(\mathrm{PRM})}=\alpha_{0}+\alpha_{1} \text { PRM }_{\mathrm{t}-{ }^{-1}} \\
\text { PRODM }_{\mathrm{t}}= & \mathrm{g}_{(\mathrm{PRM})}=\pi_{0}+\alpha_{1} \text { PRM }_{\mathrm{t}-1}{ }^{-1}
\end{array}
$$

en la cual, para el t-ésimo año, $\alpha_{1}$ encierra el efecto de $\operatorname{PSEM}_{t}$ e $\mathrm{iN}_{\mathrm{t}-2} ; \alpha_{0}$ encierra el efecto de LLUVIA $_{t}$

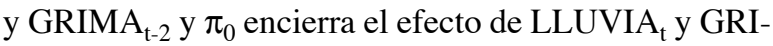
$\mathrm{MA}_{\mathrm{t}-3}$. De donde, $\mathrm{f}_{(\mathrm{PRM})}$ es la oferta de maíz al productor en el escenario con cambio tecnológico y $\mathrm{g}_{(\mathrm{PRM})}$ es la oferta de maíz al productor en el escenario sin cambio tecnológico.

\section{Medición de los beneficios sociales}

Los cambios en los excedentes de productores se estimaron con rudimentos geométricos y de cálculo integral. Para cada año del período estudiado, se tomaron integrales definidas de las funciones de oferta con y sin cambio tecnológico. Por su parte, la estimación del ahorro de divisas se hizo siguiendo la notación de la Figura 1; éste se estimó como la diferencia Q2 - Q1, en donde, Q2 es la oferta predicha con la función del escenario con cambio tecnológico y Q1 es la oferta predicha con la función del escenario sin cambio tecnológico.

Para derivar la parte de los beneficios sociales que es atribuible al trabajo del ICT A, se asumió que la mitad de los mismos se puede considerar como reponsabilidad del ICT A, la otra mitad resulta de la investigación privada, trabajo de extensión, transferencia de información de vendedores de insumos y de otros agentes que introduzcan innovaciones tecnológicas. Este supuesto resulta bastante restrictivo, ya que el programa del ICTA es el único con cobertura nacional, y por otra parte, el énfasis de la extensión agrícola se vino a dar a finales del período evaluado (1987-90) con el Proyecto de Generación y Transferencia de Tecnología Agropecuaria y Producción de Semillas (PROGETTAPS), sin embargo, en ausencia de información que permita separar las responsabilidades en el incremento de los excedentes de productores y ahorro de divisas, este supuesto resulta útil para hacer una primera estimación de tales beneficios sociales.

\section{Indicadores de eficiencia económica}

Para medir la rentabilidad social de la inversión en investigación agrícola, los indicadores empleados fueron la relación Beneficio/Costo $(\mathrm{B} / \mathrm{C})$ y la Tasa Interna de Retorno (TIR). Para estimar la relación B/C se utilizó una tasa de descuento del 12,5\% anual, la cual de acuerdo con Gittinger (1987) puede emplearse como la tasa del costo de oportunidad del capital en países en desarrollo.

\section{Los datos}

La información empleada se obtuvo del ICTA, el Banco de Guatemala, el Instituto Nacional de Estadística (INE) y del Instituto de Comercialización Agrícola (INDECA).

\section{RESULTADOS}

\section{Resultados estadísticos}

En el Cuadro 1 se presenta el modelo econométrico del mercado de maíz. Puede apreciarse en este cuadro que la ecuación de la oferta de maíz al productor presenta un coeficiente de determinación múltiple de 0,9235, el cual indica que el 92,35 \% de la variación de la oferta a nivel de productor lo explica esta ecuación. Además, presenta una F calculada de 32.216 que es altamente significativa, lo cual indica que como relación global, esta ecuación de regresión es significativamente explicativa de la oferta de maíz. Por otra parte, de acuerdo con el criterio de Theil (1971) $|t|>1$, el precio relativo semilla/maíz $\left[\mathrm{PSEM}_{\mathrm{t}} / \mathrm{PRM}_{\mathrm{t}-1}\right]$, los gastos acumulados en investigación en maíz $\left[\mathrm{GR}_{\mathrm{M}} \mathrm{MA}_{\mathrm{t}-2}\right.$ ] y la precipitación pluvial [LLUVIA ${ }_{\mathrm{t}}$ ], registran efectos significativos en la oferta de maíz. Respecto al análisis de autocorrelación, el estadístico de Durbin- Watson registra un valor de 2.053, el cual es mayor que el límite menor (dL) que dan las tablas para este coeficiente, con lo cual se pueden descartar la posible existencia de autocorrelación positiva. Este estadístico en cuestión, también es menor que cuatro menos el límite superior que dan las tablas para este coeficiente (4 - dU), lo cual también pemiten descartar la posible existencia de autocorrelación negativa. 
Cuadro 1. Modelo econométrico del mercado de maíz, en Guatemala.

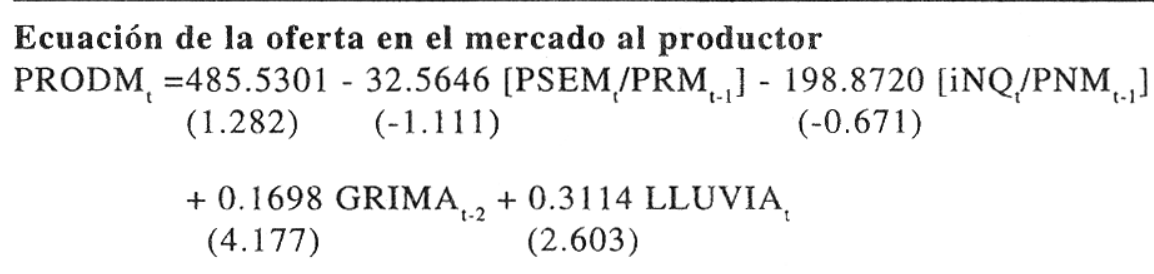

$\mathrm{R}^{2}:$ 0,9235; Fc: 32.216 [0.0001]; Durbin-Watson: 2.053

Identidad de la demanda en el mercado de consumo

$\mathrm{QDCM}_{\mathrm{t}}=\mathrm{PRODM}_{\mathrm{t}}+\mathrm{MM}_{\mathrm{t}}-\mathrm{XM}_{\mathrm{t}}$

Identidad del precio al productor

$\mathrm{PRM}_{\mathrm{t}}=\mathrm{PIM}_{\mathrm{t}}$

Identidad del saldo de comercio

$\mathrm{SCM}_{\mathrm{t}}=\mathrm{PRODM}_{\mathrm{t}}-\mathrm{QDCM}_{\mathrm{t}}$

NOTA: Los valores entre paréntesis son las razones de " $\mathrm{t}$ " de Student, el valor entre corchetes es la significancia de $\mathrm{F}$ de Fisher; y R2 es el coeficiente de determinación múltiple. Los valores límites de Durbin-Watson al $1 \%$ de probablidad para $\mathrm{n}=16$ y cuatro variables explicativas, para probar ausencia de autocorrelación positiva son: $\mathrm{dL}=0,53 \mathrm{y} \mathrm{dU}=$ 1,66; y para probar ausencia de autocorrelación negativa son: 4 - $d U=2,34$ y 4 - $d \mathrm{~L}=3,47$

en donde:

Variables endógenas:

PRODM Oferta de maíz en el mercado al productor (miles $t$ );

QDCM Cantidad demandada de maíz en el mercado de consumo (miles t);

PRM Precio real de maíz al productor (Quetzales/t);

SCM Saldo de comercio de maíz (miles t);

Variables predeterminadas:

PRMt-1 Precio real esperado de maíz al productor. Precio del año anterior (Quetzales/t);

PNMt-1 Precio nominal esperado de maíz al productor. Precio del año anterior (Quetzales/kg);

PSEM Precio real de la semilla mejorada de maíz (Quetzales/t);

iNQ Costo nominal de los intereses de un Quetzal (Quetzales)

GRIMAt-2 Conocimiento técnico o factor de cambio tecnológico. Medido a través de los gastos reales de investigación en el maíz acumulados con dos años de rezago (miles Quetzales);

LLUVIA Precipitación pluvial anual $(\mathrm{mm})$;

MM Importaciones de maíz (Quetzales/t);

XM Exportaciones de maíz (miles $\mathrm{t}$ );

PIM Precio CIF real de importación de maíz (Quetzales/t);

En el Cuadro 2 se presentan los resultados de un diagnóstico de multicolinealidad practicado a la ecuación de oferta de maíz, el cual se realizó con componentes principales. En este análisis se considera que existe multicolinealidad si a la vez que existe un alto número de condición un componente contribuye a explicar gran parte de la varianza de más de una variable. Como puede apreciarse en este cuadro, no existen signos serios de multicolinealidad, sin embargo, el hecho de que el mismo componente que explica la mitad de la varianza de $\left[\mathrm{PSEM}_{\mathrm{t}} / \mathrm{PRM}_{\mathrm{t}-1}\right]$ también explica la mitad de la varianza de $\left[\mathrm{iNQ} / \mathrm{PNM}_{\mathrm{t}-1}\right]$, sugiere la existencia de alguna colinealidad entre estos precios relativos.
Para determinar la seriedad de esta colinealidad, se ajustaron dos modelos de oferta de maíz, en los cuales se quitó y se adicionó altemamente el efecto de estos precios relativos. Este análisis es puramente intuitivo y se condujo comparando los regresores y errores estándard de estos modelos con los estimados con el primer modelo (llamémosle el modelo completo). Estas comparaciones se presentan en el Cuadro 3. Puede apreciarse en este cuadro, que al quitar altemamente cada uno de estos precios relativos, la variabilidad de los regresores (sus errores estándard) se reduce ligeramente, 10 cual corrobora la existencia de una ligera colinealidad (de acuerdo con la literatura, en presencia de multicolinealidad, los 
Cuadro 2. Diagnóstico de multicolinealidad, en el modelo de oferta de maíz al productor, en Guatemala.

\begin{tabular}{|c|c|c|c|c|c|c|c|}
\hline \multirow{2}{*}{$\begin{array}{l}\text { Componente } \\
\text { principal }\end{array}$} & \multirow{2}{*}{$\begin{array}{c}\text { Raíces } \\
\text { Características }\end{array}$} & \multirow{2}{*}{$\begin{array}{l}\text { Número de } \\
\text { Condición }\end{array}$} & \multicolumn{5}{|c|}{ Varianza de la J-Esima variable explicada por el I-Esimo compononte principal } \\
\hline & & & Intercepto & {$\left[\mathrm{PSEM} / \mathrm{PRM}_{t-1}\right]$} & {$\left[\mathrm{iNQ}_{\mathrm{t}} / \mathrm{PNM}_{\mathrm{t}-1}\right]$} & GRIMA $_{t-2}$ & Lluvia $_{\mathrm{t}}$ \\
\hline 1 & 4,5978 & 1,0000 & 0,0001 & 0,0009 & 0,0005 & 0,0028 & 0,0004 \\
\hline 2 & 0,3681 & 3,5340 & 0,0001 & 0,0021 & 0,0059 & 0,1712 & 0,0000 \\
\hline 3 & 0,0204 & 15,0085 & 0,0052 & 0,3947 & 0,0089 & 0,1044 & 0,1914 \\
\hline 4 & 0,0117 & 19,7898 & 0,0001 & 0,5058 & 0,5033 & 0,3309 & 0,0831 \\
\hline 5 & 0,0019 & 49,6782 & 0,9945 & 0,0965 & 0,4814 & 0,3907 & 0,7251 \\
\hline
\end{tabular}

NOTA: Se dice que existe multicolinealidad, si simultaneámente con un alto número de condición un componente principal contribuye a explicar una alta proporción de la varianza de más de una variable.

Cuadro 3. Comparación de los regresores y errores estandard, en modelos de oferta de maíz al productor en Guatemala.

\begin{tabular}{|c|c|c|c|}
\hline Variables & Modelo complet & $\begin{array}{l}\text { Modelo donde falta } \\
{\left[\text { iNQ }_{t} / \mathrm{PNM}_{\mathrm{t}-1}\right]}\end{array}$ & $\begin{array}{l}\text { Modelo donde falta } \\
{\left[\text { PSEM }_{t} / \text { PRM }_{t-1}\right]}\end{array}$ \\
\hline \multicolumn{4}{|c|}{ COEFICIENTES DE REGRESION PARCIAL } \\
\hline Intercepto & 485,5301 & 310,4776 & 332,8169 \\
\hline [PSEMt/PRMt-1] & $-32,5646$ & $-37,0013$ & \\
\hline [iNQt/PNMt-1] & $-198,8720$ & & $-273,2227$ \\
\hline GRIMAt-2 & 0,1698 & 0,1927 & 0,1685 \\
\hline LLUVIAt & 0,3114 & 0,3392 & 0,3374 \\
\hline \multicolumn{4}{|c|}{ ERRORES ESTANDARD DE LOS COEFICIENTES DE REGRESION PARCIAL } \\
\hline Intercepto & 378,7144 & 2680107 & 356,3056 \\
\hline [PSEMt/PRMt-1] & 29,3150 & 27,8961 & \\
\hline [iNQt/PNMt-1] & 296,5611 & & 291,7127 \\
\hline GRIMAt- 2 & 0,0407 & 0,0214 & 0,0410 \\
\hline LLUVIAt & 0,1196 & 0,1097 & 0,1185 \\
\hline \multicolumn{4}{|c|}{ COEFICIENTES DE DETERMINACION MULTIPLE } \\
\hline & 0,9235 & 0,9204 & 0,9150 \\
\hline
\end{tabular}

errores estándar tienden a ser más grandes; Gujarati 1982, Johnston, 1979). Por otra parte, puede apreciarse que las magnitudes de los regresores, exceptuando el de $\left[\mathrm{iNQ}_{\mathrm{t}} / \mathrm{PNM}_{\mathrm{t}-1}\right]$, registran leves variaciones al quitar o adicionar estos precios relativos, lo cual sugiere que la colinealidad no es perjudicial para estos regresOres. Ahora, el hecho de que el regresor de $\left[\mathrm{iNQ}_{\mathrm{t}} / \mathrm{PNM}_{\mathrm{t}-1}\right]$ se incremente en valor absoluto en casi $50 \%$ al quitar $\left[\mathrm{PSEM}_{\mathrm{t}} / \mathrm{PRM}_{\mathrm{t}-1}\right]$, pero que todavía se mantenga no significativo (su error estándar es mayor que su regresor, lo cual genera una $\mathrm{t}<\mathrm{l}$ ) y dentro de los límites que establece un error estándar arriba y debajo del regresor del modelo completo, indica que la ausencia de significancia no se debe a la colinealidad, por lo que desde la pers- pectiva de esta variable, la ligera colinealidad identificada en el Cuadro 2, tampoco resulta perjudicial, por lo que el modelo completo puede emplearse para los usos predictivos en que fue ocupado en este estudio.

\section{Resultados económicos}

En el Cuadro 4 se presentan los incrementos en los excedentes de productores y los ahorros de divisas resultantes de las decisiones de continuar anualmente el programa de investigación en maíz y el $50 \%$ de tales beneficios que es adjudicable al trabajo del ICTA en este cultivo. 
Cuadro 4. Beneficios sociales de la investigación agrícola en maíz, en Guatemala 1973 - 90 (miles de Quetzales de 1970)

\begin{tabular}{ccccc}
\hline Año & $\begin{array}{c}\text { Incremento en exceden- } \\
\text { te de productores }\end{array}$ & $\begin{array}{c}\text { Ahorro de } \\
\text { divisas }\end{array}$ & $\begin{array}{c}\text { Beneficios sociales } \\
\text { totales }\end{array}$ & $\begin{array}{c}\text { Beneficios sociales } \\
\text { Generados por ICTA* }\end{array}$ \\
\hline 1973 & 0 & 0 & 0 & 0 \\
1974 & 0 & 0 & 0 & 0 \\
1975 & 0 & 0 & 0 & 0 \\
1976 & 0 & 0 & 0 & 0 \\
1977 & 867348 & 1102852 & 1970200 & 985100 \\
1978 & 1910103 & 1755293 & 3665396 & 1832698 \\
1979 & 2527271 & 2658951 & 5186222 & 2593111 \\
1980 & 3207267 & 2992309 & 6199576 & 3099788 \\
1981 & 2798827 & 2938408 & 5737235 & 3453391 \\
1982 & 2455472 & 4451311 & 6906783 & 2833961 \\
1983 & 2787954 & 2879968 & 5667922 & 2315972 \\
1984 & 2045049 & 2586895 & 4631944 & 1379678 \\
1985 & 1352781 & 1406575 & 2759356 & 1890606 \\
1986 & 1729739 & 2051472 & 3781211 & 1775413 \\
1987 & 1353729 & 2197098 & 3550827 & 1667771 \\
1988 & 1515785 & 1819758 & 3335543 & 3219984 \\
1989 & 2390747 & 4049221 & 6439968 & 2126816 \\
1990 & 2134537 & 2119095 & 4253632 & \\
\hline
\end{tabular}

* Se asume que el $50 \%$ el total de beneficios sociales es responsabilidad del ICTA.

En el Cuadro 5 se presentan los flujos de beneficios sociales y gastos de investigación, y los indicadores de eficiencia económica estimados para medir la rentabilidad social. Puede apreciarse en este cuadro que el programa de investigación en maíz registró una relación B/C de 6.055 y una TIR de $96.948 \%$, los cuales evidencia que este programa tiene una alta rentabilidad social para la economía nacional. La relación $\mathrm{B} / \mathrm{C}$ indica que por cada Quetzal gastado en investigación agrícola en maíz retornaron 6.06 en beneficios sociales. Por su parte, la TIR indica que si el programa de investigación agrícola en maíz fuese una actividad privada y las ganancias sociales fuesen beneficios privados, este programa pudo haberse financiado con préstamo y pagar intereses hasta por $96.948 \%$ anual y no incurrir en pérdida. Otra forma de interpretar la TIR es que para generar un flujo de beneficios iguales a los generados por este program,a de investigación, los gastos de investigación debieron haberse depositado en una cuenta de ahorro y cobrar una tasa de interés anual de $96.948 \%$ anual durante 18 años.

Según Gittinger (1987), el costo de oportunidad del capital en los países en desarrollo se encuentra entre el 8 y $15 \%$, por lo que el hecho de que la TIR estimada para este programa sea seis veces más grande que el límite superior del costo de oportunidad del capital, es otro elemento qué evidencia la alta rentabilidad de este programa.

\section{IMPLICACIONES}

1. De acuerdo con Evenson y Flores (1978), los altos índices de rentabilidad sugieren que el nivel de inversión en investigación agrícola ha sido reducido. Este argumento es equivalente a indicar que si los beneficios sociales son el resultado de asignar recursos a una función de producción, la asignación de recursos para investigación agrícola en maíz, todavía se encuentra en la primera etapa de la producción, pues en esta etapa, el nivel de recursos empleados es bajo y registra productividades altas y crecientes, lo cual se asemeja al perfil mostrado por la investigación en maíz. Por tanto, una primera implicación de este trabajo es que la asignación de recursos en este programa de investigación no ha sido la óptima.

2. Por otra parte, los bajos niveles de inversión en investigación agrícola en maíz y las altas ganancias sociales obtenidas, sugieren también que la sociedad está ganando una cuasi renta, la cual de acuerdo con la teoría económica debería ser empleada en el largo plazo para reproducir el recurso que la genera. En este caso, esta cuasi renta debe utilizarse para reproducir la capacidad investigativa del ICTA, tanto en instalaciones físicas como en programas de 
Cuadro 5. Beneficios sociales, gastos e índices de rentabilidad social de la investigación agrícola en maíz a Guatemala, 1973 - 90.

\begin{tabular}{|c|c|c|}
\hline Año & $\begin{array}{l}\text { Beneficios sociales } \\
\text { generados por el ICTA } \\
\text { miles de Quetzales de } 1970\end{array}$ & $\begin{array}{c}\text { Gastos totales de } \\
\text { investigación en maíz } \\
\text { miles de Quetzales de } 1970\end{array}$ \\
\hline 1973 & 0 & 60467 \\
\hline 1974 & 0 & 120933 \\
\hline 1975 & 0 & 120933 \\
\hline 1976 & 0 & 214332 \\
\hline 1977 & 985100 & 314153 \\
\hline 1978 & 1832698 & 335618 \\
\hline 1979 & 2593111 & 323553 \\
\hline 1980 & 3099788 & 350000 \\
\hline 1981 & 2868617 & 367732 \\
\hline 1982 & 3453391 & 307873 \\
\hline 1983 & 2833961 & 208941 \\
\hline 1984 & 2315972 & 203028 \\
\hline 1985 & 1379678 & 164333 \\
\hline 1986 & 1890606 & 196366 \\
\hline 1987 & 1775413 & 258493 \\
\hline 1988 & 1667771 & 197204 \\
\hline 1989 & 3219984 & 185151 \\
\hline 1990 & 2126816 & 129258 \\
\hline Total & scontado 9100924 & 1502934 \\
\hline \multicolumn{3}{|c|}{$\mathrm{Al} 12,5 \%$ anual $*$} \\
\hline \multicolumn{3}{|c|}{ Relación beneficio/costo: 6055} \\
\hline \multicolumn{3}{|c|}{ Tasa interna de retorno: $96948 \%$} \\
\hline
\end{tabular}

formación de capital humano y para evitar el éxodo del capital humano logrado.

La investigación agrícola en maíz probó ser un instrumento para la liberación de estrangulamientos de balanza de pagos, por lo que su utilidad como instrumento de política trasciende a las políticas agrícola, alimentaria y de desarrollo rural a las que pertenece y le abre espacio para pertenecer al conjunto de instrumentos de las políticas monetaria y de comercio exterior.

\section{LITERATURA CITADA}

CHINN, D.L. 1976. The marketed surplus of a subsistence crop: Paddy Rice in Taiwan. American Journal of Agricultural Economics 58(3):583-587.

EVENSON, R. 1967. The contribution of agricultural research to production. Journal of Faryn Economics 49: 1415-1425.
EVENSON, R. 1978. "The organization of research to improve crops and animals in low-income countries". In: T.W. Schultz (ed) Distortions of agricultural Incentives. Bloomington, Indiana University Press. pp. 223-245.

EVENSON, R. E.; P. M. FLORES. 1978. "Social returns to rice research" in economic consequences of the New Rice Technologies, Los Baños, Filipinas, International Rice Research Institute (IRRI). p. 243-265.

GITTINGER, 1. P. 1987. Análisis económico de proyectos agrícolas. 2da. Reimpresión de la 2da. ed. Traducción del inglés de C. Saavedra Arce. Madrid, Tecnos.

GRILICHES, Z. 1964. Research expenditures, education, and the aggregate agricultural production Function. American Economic Review 45:419-428.

GUJARATI, D. 1982. Econometría básica. Traducción del inglés de J.M. Mesa. México, Libros McGrawHill.

JOHNSTON, J. 1979. Métodos de econometría. 2da. Reedición. Traducción del inglés de J.M. Otero Moreno. Barcelona, Vicens-Vives. 
MAR TINEZ, 1. C. y G. SAIN. 1983. The economic returns to institutional innovations in national agricultural research: On-FarmResearch in IDIAP Panamá. CIMMYTEconomics Program Working Paper 04/83. México, Centro Internacional de Mejoramietno de Maíz y Trigo (CIMMYT).

NORTON, G. W; J. S. DAVIS. 1981. Evaluating returns to agricultural research: A review. American Journal of Agricutural Economics 63(4):685-699.

REYES H, M. 1995. Factores que han contribuido al sostenimiento de la autosuficiencia de maíz en Guatemala, 1975-90: Un análisis econométrico. Publicación Miscelánea 29. Guatemala, ICTA.
SAIN, G.; R. MATUTE. 1993. "Cambio tecnológico e investigación en fincas en el Departamento de Atlántida, Honduras". In: Síntesis de resultados experimentales del PRM, Volumen 4. Guatemala, Programa Regional de Maíz de Centroamérica y el Caribe-CIMMYT. p. 198-211.

SCOBIE, G. M.; R. POSADA. 1976. The impact of high-yielding rice varieties in Latin America with special emphasis on Colombia. A preliminary repart. Cali, Colombia, CIA T.

TOQUERO, Z.; B. DUFF; T. ANDEN-LACSINA; Y. HAYAMI. 1975. Marketable surplus functions for a subsistence crop: Rice in the Philippines. American Journal of Agricultural Economics 57(4):705-709. 